\title{
EFECTO DE LA FERTILIZACIÓN NITROGENADA EN EL CAFETO CONILON SOBRE EL RENDIMIENTO Y ALGUNOS INDICADORES DE CALIDAD DE SUELOS CAMBISOLES DE CUBA
}

\section{EFEITO DO FERTILIZANTE DE NITROGÊNIO NO CAFETO CONILON NO RENDIMENTO E ALGUNS INDICADORES DE QUALIDADE DO SOLO CAMBISOL DO CUBA}

\section{Alberto Pérez Díaz ${ }^{1}$, Carlos Bustamante González ${ }^{2}$, Gloria Martín Alonso ${ }^{3}$ y Ramón Rivera Espinosa ${ }^{3}$}

${ }^{1}$ Universidad de Guantánamo, Facultad Agroforestal de Montaña. Carretera a Santiago de Cuba, Km 2 1⁄2, municipio Guantánamo, provincia Guantánamo, Cuba, CP 95 300. E-mail aperez@ fam.cug.co.cu

${ }^{2}$ Instituto de Investigaciones Agroforestales. UCTB Tercer Frente, CP 92700,

Santiago de Cuba, Cuba. E-mail bustamante@ecicc.ciges.inf.cu

${ }^{3}$ Instituto Nacional de Ciencias Agrícolas. Carretera de Tapaste, Km 3 1/2, Gaveta Postal No 1, San José de las Lajas, Mayabeque, Cuba Código Postal 32700 E-mail gloriam@inca.edu.cu, rrivera@inca.edu.cu

\section{RESUMEN}

La investigación se desarrolló durante dos ciclos productivos del cafeto con el objetivo de evaluar el efecto de 10 años de fertilización $\mathrm{N}$ sobre el rendimiento de Coffea canephora cultivado en suelos Cambisoles y algunos indicadores de la calidad del suelo $(\mathrm{pH}, \mathrm{M} . \mathrm{O}$, respiración biológica, nitrificación). Se encontró una respuesta positiva y significativa entre el rendimiento y la fertilización nitrogenada, similar en ambos sitios y ciclos $\left(\mathrm{R}^{2}>93,6 \%\right)$, lo que conllevó a plantear que, la especie canephora, puede alcanzar rendimientos entre 1,5 y 2 t.ha ${ }^{-1}$ de café oro, con dosis entre $100-200 \mathrm{~kg} \cdot \mathrm{ha}^{-1}$ de N. Las dosis de fertilizantes propuestas para alcanzar altos rendimientos, incrementaron la actividad microbiana del suelo y no afectaron la materia orgánica de los suelos. Se encontró disminución del pH del suelo respecto a su estado inicial aunque los valores de este indicador, encontrados luego de la fertilización nitrogenada, se encontraban dentro del rango establecido para el cafeto.

Palabras-clave: Fertilización Nitrogenada. Coffea canephora. Indicadores del Suelo. Respiración Biológica. Materia Orgánica

\section{RESUMO}

A investigação foi desenvolvida durante dois períodos o efeito da fertilização $\mathrm{N}$ foi avaliado no rendimento de Coffea canephora de cultivado em solo Cambisol e em alguns indicadores do solo 
(pH, M.O, respiração biológica, nitrificação). Ele era uma resposta positiva e significante entre o rendimento e fertilização nitrogenada, semelhante em lugares e ciclos $\left(\mathrm{R}^{2}>93,6 \%\right)$, o que agüentou para esboçar que, o espécies canephora, pode alcançar rendimentos entre 1,5 e 2 t.ha $^{-1}$ de café, com dose entre 100 - $200 \mathrm{~kg} \cdot \mathrm{ha}^{-1}$ de N. A doses de fertilizantes propuseram alcançar rendimentos altos, aumentou a atividade microbiana da solo e eles não afetaram o assunto orgânico da solo. Ele era diminuição do $\mathrm{pH}$ da solo que considera o estado inicial de embora os valores deste indicador, opondo depois do fertilização nitrogenada, que eles estavam dentro da gama estabelecida para o café.

Palavras-chave: Fertilização Nitrogenada. Coffea canephora. Indicadores do Solo. Respiração Biológica. Materia Orgânica.

\section{INRODUCCION}

El uso del $\mathrm{N}$ en la agricultura debe ser restringido, sujeto a criterios técnicos sobre bases científicas y económicas, por los costos elevados de los insumos y ambientales. Para el caso del cafeto, el $\mathrm{N}$ es el elemento que más influye sobre los rendimientos del cultivo (LEAL et al., 2009), en parte por las altas cantidades que del mismo requiere, así como por las escasas cantidades que aporta el suelo para garantizar los rendimientos (Da SILVA et al., 2008). A nivel internacional las dosis de $\mathrm{N}$ para el cafeto oscilan entre 80 - $412 \mathrm{~kg} \mathrm{ha}^{-1} \mathrm{y}$ dependen fundamentalmente del nivel de rendimiento esperado, de la tecnología de cultivo y de las condiciones edafoclimáticas (SADEGHIAN, 2008).

En Cuba durante los años 1986 - 1990 y 1991 - 1995 se ejecutaron experimentos de fertilización nitrogenada para plantaciones de Coffea arabica L. sembradas a altas densidades en diferentes condiciones edafoclimáticas, con el objetivo de definir: sistemas de fertilización en función del rendimiento esperado y fraccionamiento más adecuado, criterios de extracción y exportación de la plantación, la participación del $\mathrm{N}$ del fertilizante y del suelo en la nutrición de las plantas (RIVERA, 2006) quedando pendiente evaluar el efecto de la fertilización sobre los indicadores del suelo.

Al respecto, en la literatura internacional se hace referencia a parámetros biológicos con potencialidad para ser utilizados como indicadores de calidad de suelo, como son la biomasa microbiana, la respiración biológica, grupos funcionales de la microbiota, las actividades enzimáticas, la composición y diversidad de la comunidad microbiana, entre otras (GARBISU et al., 2007; SAMPAIO et al., 2011).

Los aspectos relacionados con la nutrición nitrogenada en Cuba, se han estado manejado según lo orientado para $C$. arabica, sin tener en cuenta las características propias de la especie $C$. canephora: capacidad de formar múltiples tallos, tolerancia a altas temperaturas y mayor potencial de rendimiento en áreas por debajo de los 400 m.s.n.m., lo que puede implicar un manejo nutrimental diferente. De ahí la necesidad de establecer no sólo el sistema de fertilización mineral $\mathrm{N}$ para $C$. canephora cultivado sobre suelos Cambisoles, sino su efecto sobre algunos indicadores químicos y microbiológicos del suelo.

\section{MATERIALES Y MÉTODOS}

La investigación se desarrolló durante el período de 1995 a 2007 en dos localidades de los macizos montañosos Sierra Maestra y Sagua - Nipe - Baracoa de Cuba:

Localidad Tercer Frente (sitio Cruce de los Baños): situada en el municipio Tercer Frente, macizo Sierra Maestra, a los $20^{\circ} 09^{\prime}$ lat N y $76^{\circ} 16^{\prime}$ long O, a $35 \mathrm{~km}$ ONO de la ciudad de Santiago 
de Cuba, a 150 m.s.n.m. Tipo de suelo: Pardo ócrico sin carbonatos (MINAG, 1999), que se corresponde con un Cambisol háplico (éutrico) (arcíllico) (WORLD REFERENCE BASE, 2006).

Localidad La Alcarraza: situada en el municipio Sagua de Tánamo, macizo Nipe - Sagua Baracoa, a los $20^{\circ} 35^{\prime}$ lat $\mathrm{N}$ y $75^{\circ} 15^{\prime}$ long O, a $118 \mathrm{~km}$ ESE de la ciudad de Holguín. Con una altura de 300 m.s.n.m. Tipo de suelo: Pardo gleyzoso sin carbonatos (MINAG, 1999), que se corresponde con un Cambisol estágnico (éutrico) (arcíllico) (WORLD REFERENCE BASE, 2006).

En los sitios experimentales se hicieron perfiles de suelo al inicio de los experimentos y se determinaron algunas características químicas, físicas y la clasificación de los suelos. Algunas propiedades de los suelos se presentan en la Tabla 1.

Tabla 1 - Principales características químicas del horizonte cultivable $(0-30 \mathrm{~cm})$ de los suelos bajo estudio al inicio de los experimentos.

\begin{tabular}{|c|c|c|c|c|c|c|c|c|c|}
\hline \multirow{2}{*}{ Suelos } & \multirow{2}{*}{ pH } & \multirow{2}{*}{$\begin{array}{l}\text { M.O } \\
(\%)\end{array}$} & $\mathbf{P}_{2} \mathbf{O}_{5}$ & $\mathbf{K}_{2} \mathbf{O}$ & $\mathbf{K}^{+}$ & $\mathrm{Ca}^{2+}$ & $\mathrm{Mg}^{2+}$ & $\mathrm{Na}^{+}$ & CIB \\
\hline & & & \multicolumn{2}{|c|}{$\left(\mathrm{mg.100} \mathrm{\textrm {g } ^ { - 1 } )}\right.$} & \multicolumn{5}{|c|}{$\left(\mathrm{cmol}^{-1} \mathrm{~kg}^{-1}\right)$} \\
\hline Cambisol háplico & 6.4 & 2.97 & 15.20 & 22.14 & 0.64 & 31.5 & 11.8 & 0.4 & 44.3 \\
\hline Cambisol estágnico & 5.6 & 3.07 & 16.72 & 26.0 & 0.75 & 26.8 & 10.8 & 0.27 & 38.6 \\
\hline
\end{tabular}

CIB: Capacidad de Intercambio de Bases $=\sum$ Bases cambiables.

Para el análisis del $\mathrm{pH}\left(\mathrm{H}_{2} \mathrm{O}\right)$ se utilizó el método potenciométrico, con relación suelo: solución de 1:2.5; la materia orgánica (\% M.O.) por el método de Walkley y Black; el P asimilable por extracción con $\mathrm{H}_{2} \mathrm{SO}_{4} 0.1 \mathrm{~N}$ con relación suelo: solución 1:2.5; cationes intercambiables (cmol. $\mathrm{kg}^{-1}$ ) por extracción con $\mathrm{NH}_{4} \mathrm{Ac} 1 \mathrm{Mol}^{-1}$ a $\mathrm{pH} 7$ y determinación por complejometría (Ca y $\mathrm{Mg}$ ) y fotometría de llama (K).

Los suelos Cambisoles donde se condujeron las investigaciones poseen contenidos altos de calcio y magnesio, medios de potasio y adecuada relación $\mathrm{Ca} / \mathrm{Mg}$. El pH es ligeramente ácido, y se ubicó dentro de los rangos adecuados para el cultivo del cafeto, y se presentaron valores medios de materia orgánica y fósforo (CARVAJAL, 1984).

Con el propósito de determinar el efecto de la fertilización $\mathrm{N}$ sobre el rendimiento y algunos indicadores del suelo se estudió la respuesta de cinco sistemas de fertilización nitrogenada durante dos ciclos productivos (Tabla 2) en presencia de un fondo fijo de $\mathrm{P}\left(40 \mathrm{~kg} \cdot \mathrm{ha}^{-1}\right)$ y K $\left(160 \mathrm{~kg} \cdot \mathrm{ha}^{-1}\right)$, con cuatro réplicas.

Tabla 2 - Dosis de nitrógeno $\left(\mathrm{kg}_{\mathrm{g}} \mathrm{ha}^{-1}\right)$ empleadas en los diferentes esquemas de fertilización estudiados.

\begin{tabular}{|c|c|c|c|c|c|c|}
\hline \multirow{2}{*}{ Tratamientos } & \multicolumn{3}{|c|}{ Primer ciclo productivo } & \multicolumn{3}{c|}{ Segundo ciclo productivo } \\
\cline { 2 - 7 } & $\mathbf{1 9 9 6}$ & $\mathbf{1 9 9 7}$ & $\mathbf{1 9 9 8} \mathbf{- 2 0 0 2}$ & $\mathbf{2 0 0 3}$ & $\mathbf{2 0 0 4}$ & $\mathbf{2 0 0 5}$ - 2006 \\
\hline $\mathrm{N}_{0}$ & 0 & 0 & 0 & 0 & 0 & 0 \\
\hline $\mathrm{N}_{1}$ & 30 & 45 & 50 & 50 & 75 & 100 \\
\hline $\mathrm{N}_{2}$ & 60 & 90 & 100 & 100 & 150 & 200 \\
\hline $\mathrm{N}_{3}$ & 90 & 135 & 150 & 150 & 225 & 300 \\
\hline $\mathrm{N}_{4}$ & 120 & 180 & 200 & 200 & 300 & 400 \\
\hline
\end{tabular}


La dosis de nitrógeno se fraccionó al $50 \%$, con dos aplicaciones anuales (abril e inicio de octubre). Todos los años se cosecharon los frutos maduros de cada parcela, se pesaron (kg) y se extrapolaron a t.ha ${ }^{-1}$ de café cereza y posteriormente a tonelada de café oro por hectárea (t.ha ${ }^{-1}$ de café oro), con un factor de conversión de 0,22 para el canephora en estas condiciones.

La recomendación de la dosis óptima de fertilización nitrogenada se realizó a partir de un modelo matemático donde se relacionaron los rendimientos máximos estables (RME) obtenidos en cada sitio y ciclo productivo con las dosis de $\mathrm{N}$.

Se calculó además la eficiencia agronómica (EA) para la dosis óptima propuesta en cada año y sitio experimental según la fórmula propuesta por (STEWART, 2007). Para ello se utilizaron datos de las tres últimas cosechas por ciclo productivo.

$$
\mathrm{EA}=\left(\mathrm{R}-\mathrm{R}_{0}\right) / \mathrm{D}
$$

$\mathrm{R}=$ rendimiento del cultivo con aplicación del nutriente $\left(\mathrm{kg} \cdot \mathrm{ha}^{-1}\right)$.

$\mathrm{R}_{0}=$ rendimiento del cultivo sin aplicación del nutriente $\left(\mathrm{kg} \cdot \mathrm{ha}^{-1}\right)$.

$\mathrm{D}=$ dosis del nutriente $\left(\mathrm{kg} \cdot \mathrm{ha}^{-1}\right)$.

\subsection{Influencia de la fertilización nitrogenada sobre algunos indicadores microbiológicos del suelo}

En cada parcela que constituyeron los diferentes tratamientos de fertilización nitrogenada, se seleccionaron cinco plantas y se tomaron tres muestras de suelo por cada planta en la zona de fertilización y en la profundidad de $0-30 \mathrm{~cm}$, estas muestras se mezclaron entre sí para conformar una muestra compuesta por cada parcela.

Las evaluaciones de los indicadores microbiológicos se realizaron durante los meses de octubre, noviembre y diciembre de cada año evaluado, al final de la fertilización. La respiración biológica se determinó en los años 2000 y 2001 (primer ciclo) y desde el 2004 a 2006 (segundo ciclo productivo), en ambos sitios experimentales.

Se utilizó el sistema de frasco cerrado, con humedecimiento de $25 \mathrm{~g}$ de suelo al $60 \%$ de la capacidad máxima de retención de humedad, determinada según la metodología propuesta por (FORSTER, 1995) y la determinación del $\mathrm{CO}_{2}$ se realizó al cabo de 24 horas de incubación a 30 ${ }^{\circ} \mathrm{C}$. Para determinar la respiración biológica (RB) se utilizó la fórmula descrita por (CALERO et al., 1999):

$$
\mathrm{RB}(\mathrm{mg} \mathrm{CO} / \mathrm{g} \text { suelo })=[(\mathrm{K}-\mathrm{M}) * 2.2 * \mathrm{Vt}] / \mathrm{P} * \mathrm{~V}
$$

K. ml de $\mathrm{HCl} 0.1 \mathrm{~N}$ gastado por el control M. ml de $\mathrm{HCl} 0.1 \mathrm{~N}$ gastado por la muestra

2.2. constante para cuando se trabaja con $\mathrm{HCl} 0.1$ mol. $\mathrm{L}^{-1}$ de la solución colectora para valorar. Vt. Volumen total de solución colectora $\mathrm{P}$ peso de la muestra de suelo $(\mathrm{g})$

La capacidad nitrificadora se determinó en el segundo ciclo productivo durante los años 2004, 2005 y 2006 en los dos sitios experimentales. Se utilizó el método de incubación de 25 g de suelo durante 15 días. La extracción de nitratos se realizó con $\mathrm{K}_{2} \mathrm{SO}_{4}$ al $0,01 \%$ y se utilizó ácido disulfofenólico para el desarrollo del color. Para la nitrificación potencial (NP) se adicionó sulfato de amonio al $1 \%$ respecto al peso del suelo.

La nitrificación se cuantificó a partir de la fórmula descrita por (CALERO et al., 1999):

$$
\begin{aligned}
& \mathrm{mg} \text { de } \mathrm{NO}_{3} / 100 \mathrm{~g} \text { suelo }=\underline{\mathrm{a} * \mathrm{v} * 100} *\left(\mathrm{c} / \mathrm{c}_{1}\right) \\
& \mathrm{b} * \mathrm{v}_{1}
\end{aligned}
$$

\footnotetext{
a. lectura en la curva.

100 coeficiente para llevar a $100 \mathrm{~g}$ de suelo

v. solución extractora

b. peso del suelo

$\mathrm{v}_{1}$. ml que se evaporan

c. volumen al cual se lleva la solución

$\mathrm{c}_{1}$. ml tomado para la dilución
} 


\subsection{Influencia de la fertilización $\mathbf{N}$ sobre algunos indicadores químicos $\mathbf{y}$ físico - químicos del suelo}

Se determinó el efecto de la fertilización nitrogenada sobre el pH y el porcentaje de materia orgánica del suelo en todos los tratamientos. Las evaluaciones se realizaron en diciembre de cada año, excepto el muestreo inicial que se hizo en marzo de 1996, antes del montaje del experimento. Las muestras se tomaron en la banda de fertilización de los cafetos y en la profundidad de 0 - 20 cm. En Tercer Frente las evaluaciones del pH se realizaron en los años 1996, 1999, 2001 y 2006 , mientras que en La Alcarraza se hicieron en los años 2001, 2005 y 2006. El contenido de materia orgánica se evaluó en los años 1996, 1998, 2001 y 2006 en los dos sitios.

2.3. Análisis estadístico: Se realizó un análisis de varianza a los datos obtenidos. En los casos en que se encontraron diferencias significativas entre los tratamientos, las medias fueron comparadas por la prueba de Rangos Múltiples de Duncan $(\mathrm{p}<0.05)$. Se establecieron regresiones entre los indicadores de actividad biológica evaluados en función de las dosis de $\mathrm{N}$ y el $\mathrm{pH}$ del suelo encontrado en cada uno de los tratamientos y analizados de forma conjunta todos los años.

\section{RESULTADOS Y DISCUSIÓN}

Un aspecto de alto valor metodológico y práctico para la recomendación de fertilizantes nitrogenados en el cafeto, fue la relación positiva encontrada entre las dosis óptimas de $\mathrm{N}$ y los rendimientos máximos estables anuales, para las diferentes cosechas en ambos ciclos productivos $\left(\mathrm{R}^{2}>93,65 \%\right)$, lo que indicó que el esquema de recomendación de dosis de fertilizante $\mathrm{N}$, en función de los rendimientos máximos anuales, es válido a su vez para cualquiera de los dos sitios y ciclos, y sugiere su extrapolación para condiciones de plantaciones de canephora sobre suelos Cambisoles (Figura 1).

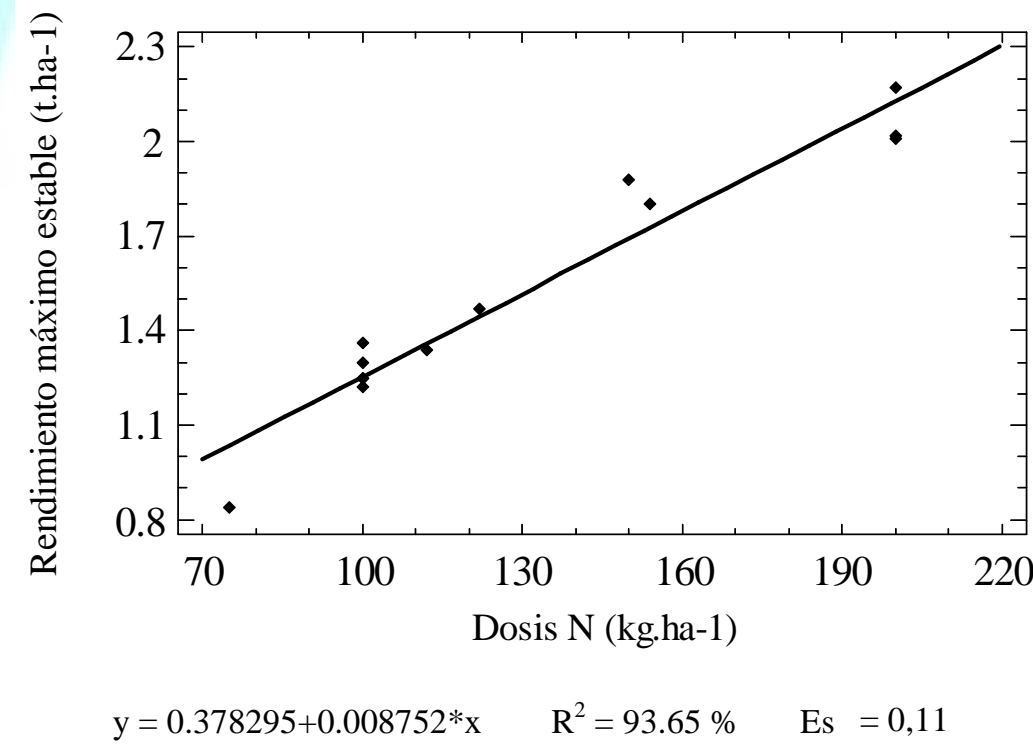

Figura 1. Relación entre dosis óptima de $\mathrm{N}$ y los rendimientos máximos estables obtenidos en ambos sitios estudiados durante los dos ciclos productivos analizados. (Cosechas 1999-2002 y 2004-2007). 
La situación encontrada en estos sitios, conlleva a plantear que, la especie canephora, puede alcanzar rendimientos entre 1,5 y 2 t.ha ${ }^{-1}$ de café oro, con independencia de la localidad y tipo de suelo, con dosis entre 100 - $200 \mathrm{~kg} \cdot \mathrm{ha}^{-1}$ de N. Un aspecto que debe quedar claro para entender adecuadamente las relaciones entre rendimiento máximo anual, fertilización nitrogenada y precipitaciones, es el hecho de que el rendimiento del cafeto depende de la variedad, cantidad de ramas nuevas en el momento de la floración, densidad de plantación, nivel de precipitaciones, intensidad de la cosecha anterior, tipo de suelo y relieve (CARVAJAL, 1984). Estas variables predeterminan un nivel de rendimiento, y la fertilización permitirá alcanzar este.

Los estimados del índice de eficiencia agronómica (Tabla 3) mostraron valores superiores en La Alcarraza que en el sitio Tercer Frente, de forma tal que dosis similares de fertilizante $\mathrm{N}$ garantizaron rendimientos mayores en La Alcarraza, con incrementos entre $10-20 \%$, con excepción del año 2001, en que el índice de eficiencia agronómica fue 2,5 veces mayores.

Tabla 3 - Eficiencia agronómica del cafeto durante dos ciclos productivos en ambas localidades (kg incremento rendimiento. $\mathrm{kg}^{-1} \mathrm{~N}$ aplicado).

\begin{tabular}{|c|c|c|c|c|}
\hline \multirow{2}{*}{ Año } & \multicolumn{2}{|c|}{ Tercer Frente } & \multicolumn{2}{c|}{ La Alcarraza } \\
\cline { 2 - 5 } & Eficiencia & RME & Eficiencia & RME \\
\hline \multicolumn{4}{|c|}{ Primer ciclo } \\
\hline $\mathbf{2 0 0 0}$ & 3,90 & 1,25 & 4,88 & 1,34 \\
\hline $\mathbf{2 0 0 1}$ & 3,10 & 1,30 & 8,65 & 1,88 \\
\hline $\mathbf{2 0 0 2}$ & 4,10 & 1,36 & 5,41 & 1,47 \\
\hline & \multicolumn{5}{|c|}{ Segundo ciclo } \\
\hline $\mathbf{2 0 0 5}$ & 7,80 & 2,18 & 4,60 & 1,22 \\
\hline $\mathbf{2 0 0 6}$ & 4,10 & 1,25 & 6,15 & 2,02 \\
\hline $\mathbf{2 0 0 7}$ & 6,50 & 1,80 & 5,65 & 2,02 \\
\hline
\end{tabular}

RME. Rendimiento máximo estable (t.ha ${ }^{-1}$ de café oro)

En el segundo ciclo se comportaron de forma similar en ambos sitios, obteniéndose siempre los mayores valores de eficiencia con altos rendimientos. En el caso de La Alcarraza, el rango de eficiencia agronómica anual encontrado fue parecido en ambos ciclos del cultivo, asociado con el hecho de que tanto el régimen de precipitación como el rango de rendimientos máximos obtenidos para cada ciclo de crecimiento fueron respectivamente similares.

\subsection{Influencia de la fertilización $\mathbf{N}$ sobre algunos indicadores químicos, físico - químicos y microbiológicos del suelo}

\subsection{1. pH}

Como tendencia se encontró disminución del pH del suelo respecto a su estado inicial en la medida que se incrementaron las dosis de nitrógeno por año, la cual se acentuó con la aplicación de 400 kg.ha ${ }^{-1}$ de N, hasta alcanzar valores de 4,52 - 4,60 en ambos sitios experimentales (Figuras 2 y 3 ). 


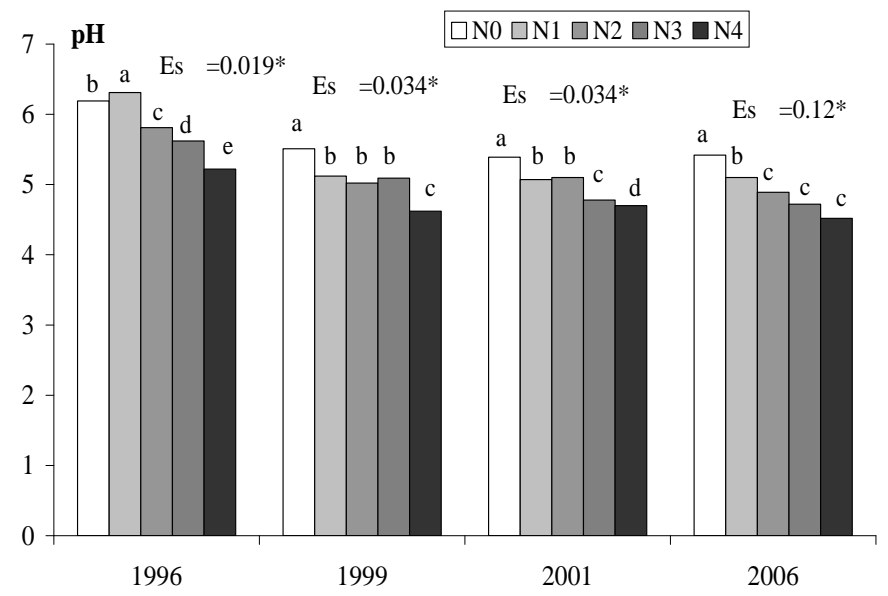

Dosis aplicadas por año

\begin{tabular}{|c|c|c|c|c|}
\hline kg.ha ${ }^{-1} \mathrm{~N}$ & 1996 & 1999 & 2001 & 2006 \\
\hline $\mathrm{N}_{0}$ & 0 & 0 & 0 & 0 \\
\hline $\mathrm{N}_{1}$ & 30 & 50 & 50 & 100 \\
\hline $\mathrm{N}_{2}$ & 60 & 100 & 100 & 200 \\
\hline $\mathrm{N}_{3}$ & 90 & 150 & 150 & 300 \\
\hline $\mathrm{N}_{4}$ & 120 & 200 & 200 & 400 \\
\hline
\end{tabular}

Figura 2. Efecto de la fertilización nitrogenada sobre el pH del suelo. Sitio Tercer Frente. Medias con letras iguales no difieren significativamente según Duncan $(\mathrm{p}<0.05)$.

Similar comportamiento de disminución del pH por efecto de la fertilización nitrogenada fue encontrado en suelos de 25 municipios cafetaleros colombianos (SADEGHIAN et al., 2006). En coincidencia, estudios realizados sobre las características de los suelos Cambisoles en diferentes agroecosistemas, encontraron mayor acidez en cafetales fertilizados, tendencia que fue relacionada con una dosis de nitrógeno de 300 - 400 kg.ha ${ }^{-1}$ (BUSTAMANTE et al., 2011).

Esto se debe a que los fertilizantes nitrogenados generan un excedente de $\mathrm{H}^{+}$que gradualmente reemplaza a las bases, que son lavadas y transportadas a los horizontes subyacentes, acompañados de aniones en las aguas de percolación (LEAL et al., 2009).

No obstante, los valores encontrados de $\mathrm{pH}$ con el sistema recomendado de fertilización $\mathrm{N}$ para altos rendimientos $\left(100-200 \mathrm{~kg} \cdot \mathrm{ha}^{-1}\right)$ en los dos ciclos productivos (BUSTAMANTE et al., 2011), fueron de 5 - 5,81 (ligeramente ácido) y se encontraban dentro del rango establecido para el cafeto, entre 5 - 6,5 (CARVAJAL, 1984), no siendo por tanto un problema la aplicación de estas dosis.

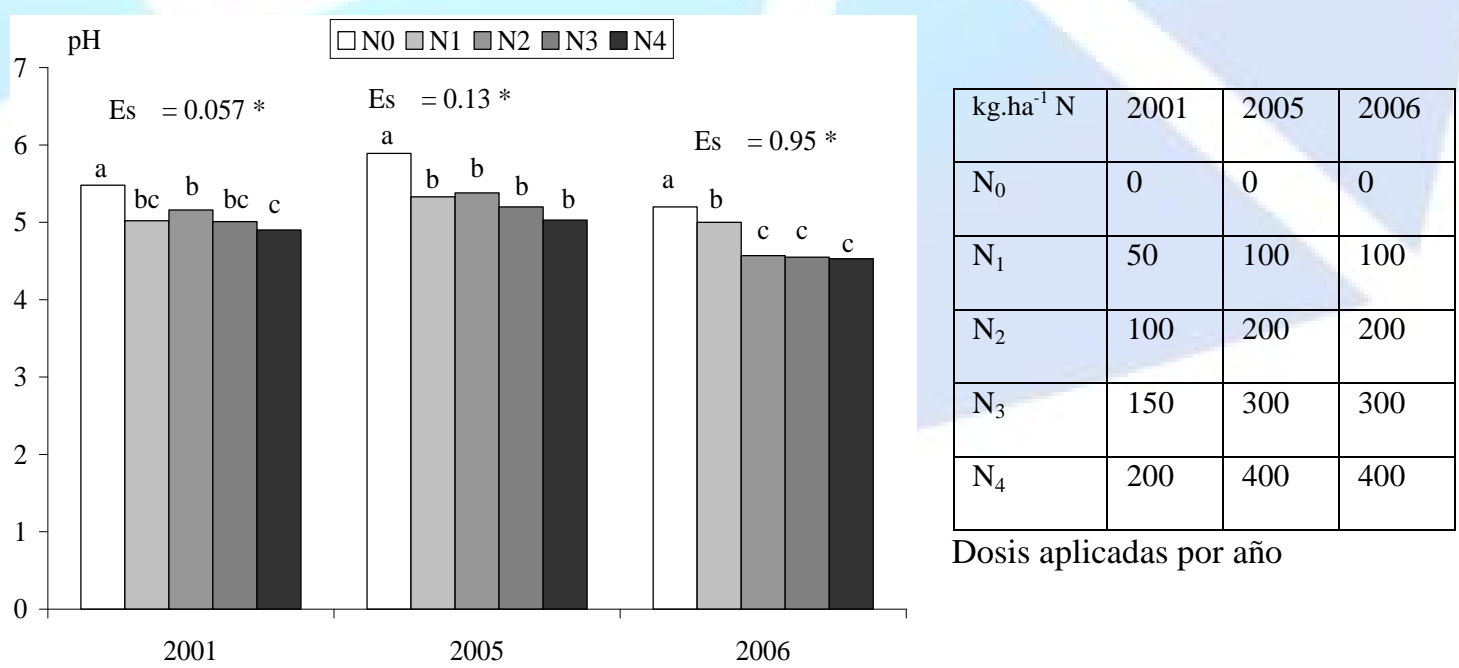

Figura 3. Efecto de la fertilización nitrogenada sobre el pH del suelo. Sitio La Alcarraza. Medias con letras iguales no difieren significativamente según Duncan $(\mathrm{p}<0.05)$. 


\subsection{La materia orgánica del suelo}

En los dos sitios no se observaron efectos negativos de la fertilización sobre la materia orgánica durante los años evaluados, sólo se encontraron incrementos de los contenidos de esta variable respecto al inicio de la investigación en 1996 (Figura 4). Esto pudo estar influenciado por el aporte de hojarasca que realizan, no sólo el cafeto sino también los árboles leguminosos que se utilizan como sombras, lo que permite que en los agroecosistemas cafetaleros existan suficientes reservas energéticas para mantener la actividad microbiana del suelo.

Respecto a esto, en Colombia, con la aplicación de $240 \mathrm{~kg} \cdot \mathrm{ha}^{-1}$ de urea, no se encontró cambios en la materia orgánica a través del tiempo. En Cuba, no encontraron efecto negativo de la fertilización nitrogenada a base de urea sobre las propiedades químicas y físicas de un suelo Pardo ócrico sin carbonatos en un cafetal bajo sombra, durante su primer ciclo productivo (BUSTAMANTE et al., 2011).

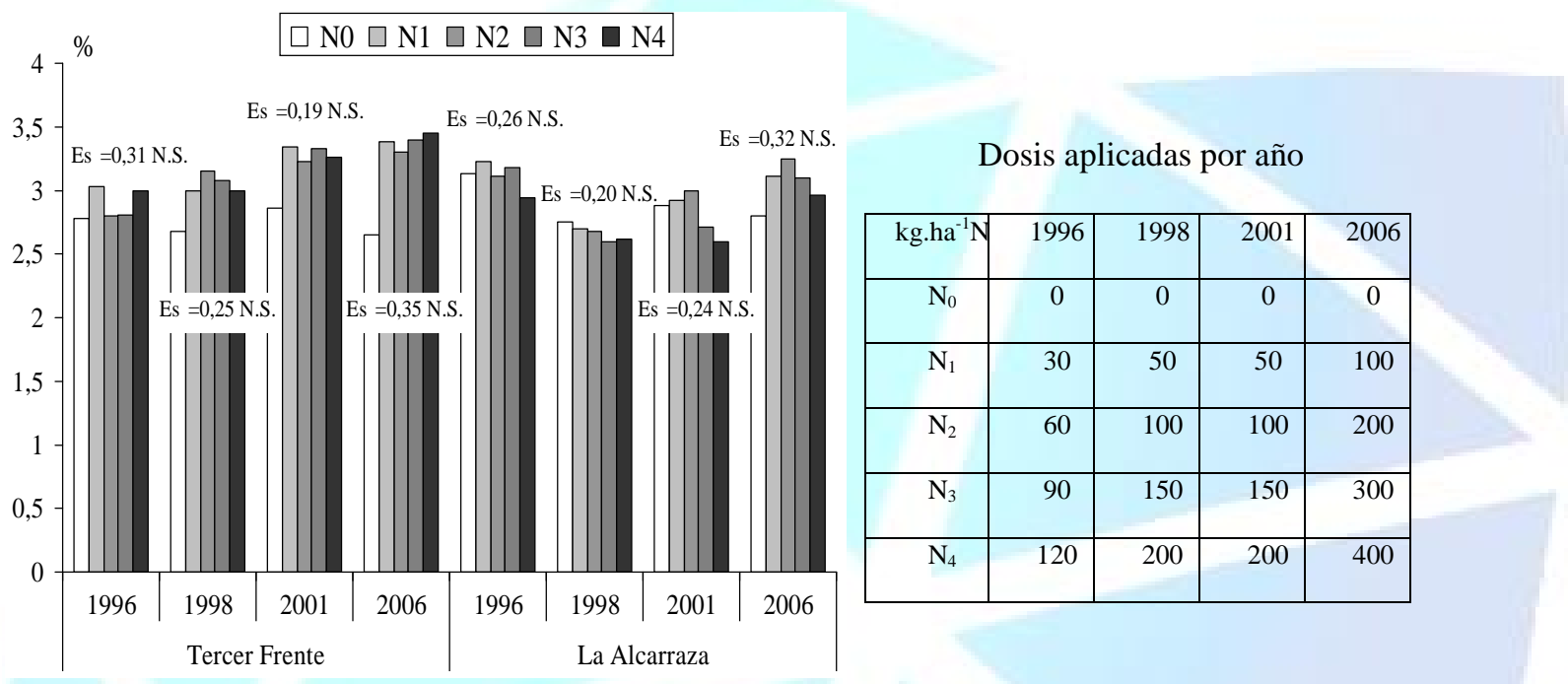

Figura 4. Efecto de las dosis de $\mathrm{N}$ en la materia orgánica (\%) del suelo. Medias con letras iguales no difieren significativamente según Duncan $(\mathrm{p}<0.05)$

\subsection{La respiración biológica del suelo}

Al valorar el efecto de la fertilización nitrogenada sobre la respiración biológica del suelo de los diferentes sitios experimentales, se encontró que esta actividad se vio favorecida con la aplicación de dosis de N entre 100 - $150 \mathrm{~kg} \mathrm{ha}^{-1}$ de N (Figura 5). Se ha observado que, la presencia de determinadas cantidades de nitrógeno mineral en el suelo, permite altos niveles de descomposición del material carbonado, al posibilitar una adecuada relación $\mathrm{C} / \mathrm{N}$ en el protoplasma microbiano, aumentando de esta manera el $\mathrm{CO}_{2}$ desprendido producto de la biodegradación microbiana (RODRÍGUEZ, 2010).

Esto pudo estar relacionado además, con el posible efecto de las dosis de $\mathrm{N}$ sobre los grupos microbianos como máximos exponentes de los procesos de degradación de la materia orgánica. En los dos sitios experimentales, se encontró además que, en la medida que las dosis de $\mathrm{N}$ aumentaron hasta $400 \mathrm{~kg} \cdot \mathrm{ha}^{-1}$ este indicador se vio deprimido.

Es importante señalar que la descomposición de las sustancias orgánicas es un proceso complejo resultante de la acción, principalmente, de hongos, bacterias y actinomicetos. No puede señalarse en la mayoría de los casos un solo organismo como causante de los cambios y aunque se postulan teorías sobre éstos, vale aclarar que han sido establecidas basadas en el comportamiento de los microorganismos en cultivos puros y sin dudas, el efecto de la población mixta de un suelo agrícola es diferente en la mayoría de los casos. 
Esta disminución de la respiración con dosis elevadas de $\mathrm{N}\left(300-400 \mathrm{~kg} \cdot \mathrm{ha}^{-1}\right.$ de $\left.\mathrm{N}\right)$ pudo deberse a que los productos ácidos derivados de la nitrificación (ácidos nitroso y nítrico) provocan rápidamente la acidificación del suelo, en grado tal, que se inhibe la respiración del suelo (RODRÍGUEZ, 2010; PÉREZ et al., 2011).

\section{Tercer Frente}

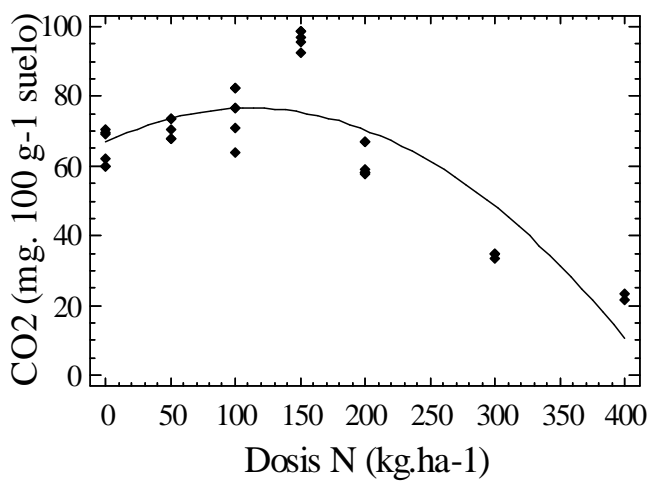

$\mathrm{y}=68,79+0,13 * x-0,00069 * x^{2}$

$\mathrm{R}^{2}=72,89 \% \quad$ Esŷ $=11,63$

\section{La Alcarraza}

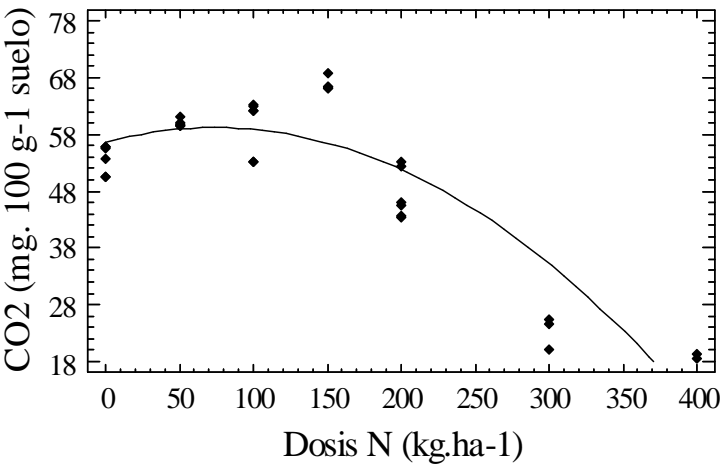

$\mathrm{y}=56,98+0,036 * \mathrm{x}-0,00038 * \mathrm{x}^{2}$

$\mathrm{R}^{2}=78,49 \% \quad$ Esŷ $=7,77$

Figura 5. Relación entre las dosis de $\mathrm{N}$ y la respiración biológica de los suelos durante 10 años.

En suelo Pardo ócrico sin carbonatos (sitio Tercer Frente) se encontró mayor actividad microbiana en la rizosfera del cafeto, asociada con una mayor respiración biológica, con relación al suelo Pardo gleyzoso sin carbonatos (sitio La Alcarraza). De esta forma, la obtención de rendimientos entre 1,22 - 1,8 t.ha ${ }^{-1}$ de café oro con la aplicación de $\mathrm{N}$ entre $100-150 \mathrm{~kg} \cdot \mathrm{ha}^{-1}$, no provocaron afectaciones en la respiración microbiana como indicadores biológicos del suelo e incluso la aplicación de $200 \mathrm{~kg} \cdot \mathrm{ha}^{-1}$ aunque disminuyó la respiración biológica respecto al tratamiento $\mathrm{N}_{1}$, esta actividad se mantuvo en valores semejantes o superiores al testigo sin fertilización.

Al valorar el efecto del $\mathrm{pH}$ sobre la respiración microbiana, se encontró relación positiva entre la respiración biológica obtenida en ambos suelos y el pH, en la medida que este disminuyó por debajo de 5, la respiración biológica se deprimió (Figura 6). Valores de pH por encima de 5,5 incrementaron la respiración biológica en ambos tipos de suelos.

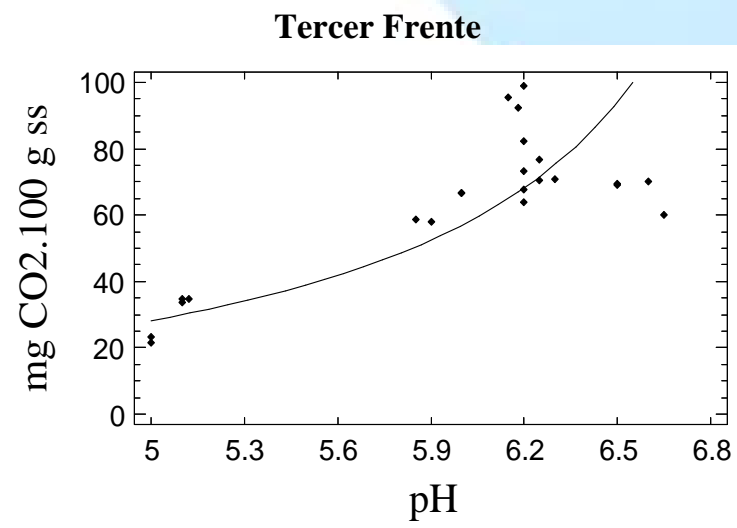

$\mathrm{y}=1 /(-0,072+0,54 / \mathrm{x})$

$\mathrm{R}^{2}=79,79 \% \quad$ Es $\hat{y}=0,0045$

La Alcarraza

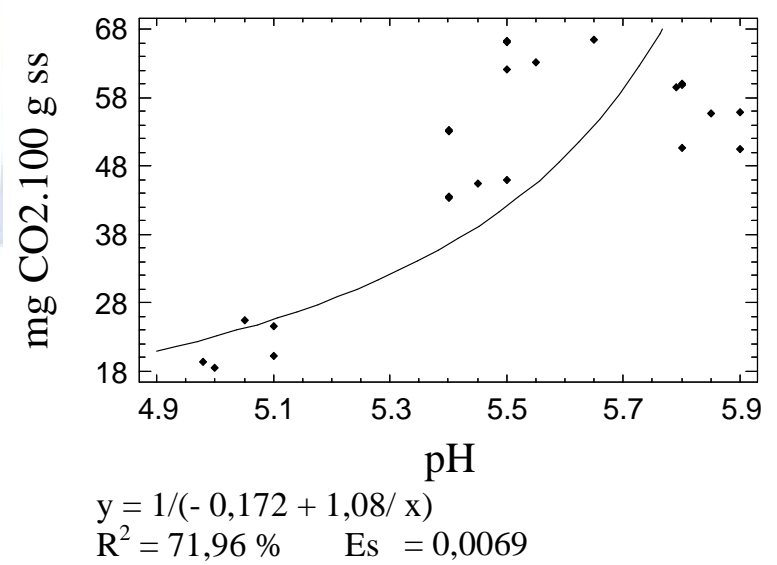

Figura 6. Relación entre el pH y la respiración biológica de los suelos durante 10 años. 
Estos resultados coinciden con lo reportado por (HEINZE et al., 2010), en el cultivo de cereales en Alemania en un suelo Cambisol Haplic fertilizado con $140 \mathrm{~kg} \cdot \mathrm{ha}^{-1}$ de $\mathrm{N}$, donde encontraron relación positiva entre el pH del suelo y la respiración biológica.

Por lo que la posible causa de variación en las actividades microbianas en la zona rizosférica del cafeto producto de la fertilización nitrogenada, estará más bien influenciada por la disminución del pH en dicha zona y por la tasa de mineralización e inmovilización del nitrógeno en el suelo, que por una disminución del carbono contenidos en la materia orgánica, como fuente de energía para su metabolismo.

\subsection{La nitrificación de los suelos}

Al analizar la actividad de nitrificación, se encontró similar tendencia en relación a las dosis de $\mathrm{N}$ propuestas para alcanzar rendimientos máximos estables de cafeto en ambos sitios experimentales, es decir, que la aplicación de $75 \mathrm{~kg} \cdot \mathrm{ha}^{-1}$ de $\mathrm{N}$ en el 2004 no afectó este indicador (Tabla 4).

En el 2005, no se encontraron diferencias significativas en la nitrificación del suelo Pardo ócrico sin carbonatos de Tercer Frente, entre las dosis $100 \mathrm{~kg} \cdot \mathrm{ha}^{-1}$ de N y $200 \mathrm{~kg}, \mathrm{ha}^{-1}$. En ese año la dosis propuesta para el sito fue de $200 \mathrm{~kg} \cdot \mathrm{ha}^{-1}$ de N. No se apreciaron diferencias significativas sobre la nitrificación entre las dosis de 200 y $300 \mathrm{~kg} \mathrm{ha}^{-1}$ de $\mathrm{N}$, y entre el testigo y la aplicación de $400 \mathrm{~kg} \mathrm{ha}^{-1}$ de N.

Sin embargo, posteriormente éste indicador se deprimió en el año 2006 y decreció con respecto al tratamiento $\mathrm{N}_{0}$ con la aplicación continuada de 300 - $400 \mathrm{~kg} \cdot \mathrm{ha}^{-1}$ de N. En el sitio La Alcarraza, a pesar de encontrarse menor cuantía de la nitrificación en el suelo Pardo gleyzoso sin carbonatos, se mantuvo igual tendencia que en el sitio Tercer Frente en relación con las dosis de N aplicadas.

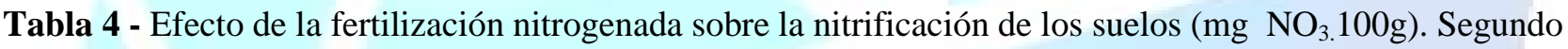
ciclo productivo.

\begin{tabular}{|l|c|c|c|c|c|c|}
\hline \multirow{2}{*}{ Dosis } & \multicolumn{3}{|c|}{ Tercer Frente } & \multicolumn{3}{c|}{ La Alcarraza } \\
\cline { 2 - 7 } & $\mathbf{2 0 0 4}$ & $\mathbf{2 0 0 5}$ & $\mathbf{2 0 0 6}$ & $\mathbf{2 0 0 4}$ & $\mathbf{2 0 0 5}$ & $\mathbf{2 0 0 6}$ \\
\hline $\mathrm{N}_{0}(0)$ & $24,63 \mathrm{c}$ & $25,73 \mathrm{c}$ & $38,80 \mathrm{~b}$ & $21,46 \mathrm{c}$ & $20,18 \mathrm{c}$ & $22,35 \mathrm{~b}$ \\
\hline $\mathrm{N}_{1}(75)$ & $52,92 \mathrm{a}$ & & & $32,45 \mathrm{~b}$ & & \\
\hline $\mathrm{N}_{1}(100)$ & & $50,50 \mathrm{a}$ & $53,80 \mathrm{a}$ & & $30,15 \mathrm{a}$ & $33,70 \mathrm{a}$ \\
\hline $\mathrm{N}_{2}(150)$ & $53,00 \mathrm{a}$ & & & $36,17 \mathrm{a}$ & & \\
\hline $\mathrm{N}_{2}(200)$ & & $47,78 \mathrm{ab}$ & $42,05 \mathrm{~b}$ & & $30,63 \mathrm{a}$ & $29,15 \mathrm{~b}$ \\
\hline $\mathrm{N}_{3}(225)$ & $35,33 \mathrm{~b}$ & & & $34,12 \mathrm{~b}$ & & \\
\hline $\mathrm{N}_{3}(300)$ & & $38,43 \mathrm{~b}$ & $24,51 \mathrm{c}$ & & $25,47 \mathrm{~b}$ & $20,17 \mathrm{c}$ \\
\hline $\mathrm{N}_{4}(300)$ & $36,85 \mathrm{~b}$ & & & $24,56 \mathrm{c}$ & & \\
\hline $\mathrm{N}_{4}(400)$ & & $24,30 \mathrm{c}$ & $21,55 \mathrm{c}$ & & $19,40 \mathrm{c}$ & $18,45 \mathrm{c}$ \\
\hline $\mathrm{ES} \times$ & $1,74^{*}$ & $3,34^{*}$ & $1,59^{*}$ & $1,25^{*}$ & $1,64^{*}$ & $1,41^{*}$ \\
\hline
\end{tabular}

Número entre paréntesis: Dosis de $\mathrm{N}$ aplicada ese año $\left(\mathrm{kg} \cdot \mathrm{ha}^{-1}\right)$. Medias con letras iguales no difieren significativamente según Duncan $(\mathrm{p}<0.05)$. 
Las variaciones encontradas en la nitrificación con las diferentes dosis de $\mathrm{N}$, pudieron ser consecuencia de la disminución del pH en la rizosfera de la planta. El pH óptimo del suelo para la respiración y desarrollo de las bacterias nitrificantes es 6,5. No obstante, estas bacterias pueden respirar lentamente en los suelos ácidos hasta $\mathrm{pH} 3,5$ y en los alcalinos, hasta un $\mathrm{pH} 10$, pero su máxima respiración y desarrollo tienen efecto en medios cercanos a la neutralidad (RODRÍGUEZ, 2010).

Los valores de nitrificación alcanzados en las dosis propuestas en el suelo Pardo ócrico sin carbonatos oscilaron entre 42 - $53 \mathrm{mg} \mathrm{NO}_{3} .100 \mathrm{~g}$ de suelo; mientras que en el suelo Pardo gleyzoso sin carbonatos alcanzaron de 29 - $33 \mathrm{mg} \mathrm{NO}_{3} .100 \mathrm{~g}$ de suelo. Al respecto, en la medida en que la nitrificación sea mayor, revelará la presencia de microorganismos capaces de llevar a cabo el proceso de conversión del amonio a nitrato de forma favorable.

Las dosis de $\mathrm{N}$ propuestas (100 - $\left.200 \mathrm{~kg} \mathrm{ha}^{-1} \mathrm{de} \mathrm{N}\right)$, no afectaron la nitrificación de los suelos, si no que favorecieron éste proceso. En general, la utilización de un conjunto mínimo de indicadores seleccionados (respiración biológica, nitrificación, materia orgánica y pH) en ambos sitios, permitió integrarse al sistema racional de recomendación de dosis $\mathrm{N}$ para el cafeto y coincidió con los criterios de valores aceptables según la tabla de interpretación que se dispone en cada indicador para los suelos Pardos de Cuba recomendada por (FONT, 2007).

\section{CONCLUSIONES}

- Se encontró una respuesta positiva y significativa entre el rendimiento y la fertilización nitrogenada, similar en ambos sitios y ciclos $\left(\mathrm{R}^{2}>93,6 \%\right)$, de forma tal que en la medida que el rendimiento máximo estable fue mayor, se incrementaron las necesidades de fertilizantes.

- Las dosis de fertilizantes propuestas para alcanzar rendimientos máximos estables en cada sitio y ciclo productivo, incrementaron la actividad microbiana del suelo.

- Las dosis propuestas, no afectaron la materia orgánica de los suelos. Se encontró disminución del pH del suelo respecto a su estado inicial aunque los valores de este indicador, encontrados luego de la fertilización nitrogenada con el sistema propuesto, se encontraban dentro del rango establecido para el cafeto.

\section{AGRADECIMIENTOS}

A los colegas de la Estación Central de Investigaciones de Café y Cacao de Cuba y el Instituto Nacional de Ciencias Agrícolas y al Centro de Estudios Ambientales de la Universidad Estadual Paulista (UNESP), por la asesoría brindada en el trabajo.

\section{REFERENCIAS}

BUSTAMANTE, C.; VIÑALS, R.; PÉREZ, A.; RODRÍGUEZ, M. Fertilización nitrogenada y uso de abonos verdes para Coffea canephora Pierre cultivado en suelos Pardos de los macizos Sierra Maestra y Nipe Sagua Baracoa. Informe Final de Proyecto Nacional. Estación Central de Investigaciones de Café y Cacao. Tercer Frente. Documento Interno, 2011. 300p.

CALERO, B.J.; GUERRERO, A.; ALFONSO, C.A.; SOMOZA, V.; CAMACHO, E. Efecto residual de la fertilización mineral sobre el estado microbiológico del suelo. La Ciencia y el Hombre, Xalapa, Ver., México, v. XI, p.89 - 94, 1999. 
CARVAJAL J.F. Cafeto, cultivo y fertilización. J. F. Carvajal-Berna: Ed. Instituto Internacional de la Potasa, Universidade do Texas, USA. 1984. 254 p.

DA SILVA, A.; ARF, O.; BUZETTI S.; DA SILVA, M. Fontes e épocas de aplicação de nitrogênio em trigo em sistema plantio direto no Cerrado. Revista Brasileira de Ciência do Solo, Número Especial, Viçosa, MG., v.32: 2717 - 2722, 2008.

FONT, L. Estimación de la calidad del suelo: criterios físicos, químicos y biológicos. Resumen de Tesis presentada en opción al grado científico de Doctor en Ciencias Agrícolas. Instituto de Suelos. La Habana, 2007. 30 p.

FORSTER, J.C. Soil physical analysis. Determination of the gravimetric water content and soil dry mass. In: Ed. Kassem, A.; Nannipieri, P. Methods in applied soil microbiology and biochemistry, Chapter 3, Elsevier Ltd., p.105 -116, 1995.

GARBISU, C.; BECERRIL, J.M.; EPELDE, L. ALKORTA, I. Bioindicadores de la calidad del suelo: herramienta metodológica para la evaluación de la eficacia de un proceso fitorremediador. Ecosistema, v. 16, p. 1697 - 2473, 2007.

HEINZE, S.; RAUPP, J.Y.; JOERGENSEN, R.G. Effects of fertilizer and spatial heterogeneity in soil $\mathrm{pH}$ on microbial biomass indices in a long-term field trial of organic agriculture. Plant Soil, South African Journal, v. 328, p. 203 - 215, 2010.

LEAL, L.; SALAMANCA, A. Y.; SADEGHIAN, S. Pérdidas de nitrógeno por volatilización en cafetales en etapa productiva. Informações Agronómicas, IPNI, Piracicaba, SP., v. 74, p. 1 - 4 , 2009.

MINAG. Nueva versión de clasificación genética de los suelos de Cuba. Instituto de Suelos. La Habana. Agrinfor. 1999. 64 p.

PÉREZ, A.; BUSTAMANTE, C.; MARTÍN, G.; RIVERA, R. Fertilización nitrogenada después de la poda del cafeto robusta en Cambisoles. Pesquisa Agropecuaria Brasileira, EMBRAPA, Brasília, DF., Brasil, v. 46 (8), p. 935-943, 2011.

RIVERA, R. Nutrición y fertilización de Coffea arabica en Cuba. 2006/ In: R. Rivera, F. Soto editores. El Cultivo del cafeto en Cuba. Investigaciones y Resultados, 2006. 500 p. ISBN 9597023-37-7.

RODRÍGUEZ, P. Compendio sobre microbiología agrícola. Departamento Agropecuario, Universidad de Oriente, 2010. 276 p.

SADEGHIAN, K.S.; GARCÍA, L.; MONTOYA J.C. Respuesta del cafeto a la fertilización con N, $\mathrm{P}, \mathrm{K}$ y $\mathrm{Mg}$ en dos fincas del departamento del Quindío. Cenicafé, Manisales (Caldas), Colombia, v. 57 (1), p. 58 - 69, 2006.

SADEGHIAN, K.S. Fertilidad del suelo y nutrición del café en Colombia: Guía Práctica. Boletín Técnico Cenicafé, Manisales (Caldas), Colombia, v. 32, p. 1 - 43, 2008. 
SAMPAIO, M.; DE-POLLI, H.; DE AQUINO, A.M.; FERNANDES, M.E.; COSTA, J. Bioindicators of soil quality in coffee organic cultivation systems. Pesquisa Agropecuaria Brasileira, 46 (5), Brasilia, mayo. 2011.

STEWART, W.M. Consideraciones en el uso eficiente de nutrientes. Informaciones Agronómicas, IPNI, Piracicaba, SP., v. 67, p.1 - 6, 2007.

WRB - World Reference Base for Soil Resources. Classification Key. FAO AGL, 2006. http://www.fao.org/ag/agl/agll/wrb/newkey.stm. <17 - 1 - 2007>. 